\title{
Corela
}

Cognition, représentation, langage

HS-34 | 2021

Les noms sous-spécifiés en français: du lexique au discours

\section{Le lexique-grammaire de noms sous-spécifiés}

Éric Laporte, Christiane Marque-Pucheu et Takuya Nakamura

\section{(2) OpenEdition \\ Journals}

Édition électronique

URL : https://journals.openedition.org/corela/13667

DOI : $10.4000 /$ corela.13667

ISSN : 1638-573X

Éditeur

Cercle linguistique du Centre et de l'Ouest - CerLICO

Référence électronique

Éric Laporte, Christiane Marque-Pucheu et Takuya Nakamura, « Le lexique-grammaire de noms sousspécifiés », Corela [En ligne], HS-34 | 2021, mis en ligne le 09 décembre 2021, consulté le 13 décembre 2021. URL : http://journals.openedition.org/corela/13667; DOI : https://doi.org/10.4000/corela.13667

Ce document a été généré automatiquement le 13 décembre 2021.

\section{(c) (i) (3) (2)}

Corela - cognition, représentation, langage est mis à disposition selon les termes de la licence Creative Commons Attribution - Pas d'Utilisation Commerciale - Partage dans les Mêmes Conditions 4.0 International. 


\title{
Le lexique-grammaire de noms sous-spécifiés
}

\author{
Éric Laporte, Christiane Marque-Pucheu et Takuya Nakamura
}

\section{Introduction}

1 Nous présentons un travail en cours de révision de tables du lexique-grammaire ${ }^{1}$ de noms prédicatifs ${ }^{2}$, visant à y découvrir les noms susceptibles d'être employés comme noms sous-spécifiés (Nss) dans des constructions syntaxiques particulières.

2 Dans cet article, nous suivons la définition du Nss donnée par Legallois $(2006,2008)$ et Legallois et Grea (2006) : schématiquement, un nom sous-spécifié fonctionne comme noyau d'un syntagme nominal servant de segment gauche d'une unité constructionnelle particulière appelée construction copulative spécificationnelle (CCS). Les exemples paradigmatiques qu'ils citent sont les suivants :

(1) a. N est que P / N, c'est que P : mon propos, c'était qu'on ne m'emmerde pas

b. $N$ est de INF / N, c'est de INF : la première mesure est de fixer la règle du je

(Legallois \& Grea $2006: 161$ )

Dans la méthodologie du lexique-grammaire, les types de noms qui apparaissent dans les cadres donnés en (1) sont décrits dans une phrase simple, considérée comme phrase canonique, et construite avec un verbe support $\left(V_{\text {sup }}\right)$ particulier. Par exemple, dans le cas de (2), le nom prédicatif $\left(N_{\text {préd }}\right)$ propos est étudié avec le $V_{\text {sup }}$ avoir dans la classe ANSU, caractérisée par une structure de phrase simple où le sujet du verbe avoir et le complément prépositionnel en sur réalisent le premier et le deuxième argument du nom, respectivement :
(2) $\mathrm{N}_{0}$ avoir Dét $\mathrm{N}_{\text {préd }}$ sur $\mathrm{N}_{1}$
$=:$ Luc a eu un propos dur sur (cette affaire + Marie) (Classe ANSU, Labelle 1974)

4 La possibilité de transformer une phrase à $V_{\text {sup }}$ du type (2) en CCS n'a pas été vérifiée. On peut donc se poser une question de recherche : est-ce qu'une phrase à $V_{\text {sup }}$ donnée peut être mise en équivalence avec une CCS, dans laquelle le segment gauche est le 
prédicat et le segment droit est son argument? Nous verrons que certaines phrases à $V_{\text {sup }}$ possèdent en effet une CCS équivalente et d'autres, non.

Pour répondre à la question de recherche posée, nous prenons comme champ d'examen une classe de phrases à $V_{\text {sup }}$ qui comportent un $N_{\text {préd }}$ du type espoir. Ce nom est étudié dans les constructions à $V_{\text {sup }}$ du type :

(3) Luc (a de l'espoir + perd l'espoir)

6 Dans ces structures, décrites dans la classe APE3 de Vivès (1983), le $V_{\text {sup }}$ de base avoir peut alterner avec ses variantes aspectuelles, telles que perdre et/ou prendre. Nous nous concentrons sur le $V_{\text {sup }}$ avoir, au détriment d'autres variantes, à cause de la nonéquivalence entre le segment gauche d'une phrase spécificationnelle et les $S N$ comportant des variantes du support avoir :

(4) L'espoir de Luc (est de partir en vacances)

$=$ L'espoir qu'a Luc (est de partir en vacances)

$\neq$ L'espoir qu'a perdu Luc (est de partir en vacances)

7 Après une présentation rapide des structures prédicat/arguments attribuables aux $N_{\text {préd }}$ de la classe APE3, nous vérifions si l'attribut phrastique d'une CCS correspond à l'un des arguments. Parmi les $N_{\text {préd }}$ permettant la reconfiguration de la phrase canonique en CSS, nous distinguerons deux types : généralement, lorsque le $N_{\text {préd }}$ est déverbal, la base verbale du premier se construit avec une complétive objet et celle du second avec une complétive sujet. Dans ce deuxième cas, la CCS qui en résulte est d'un type différent de celle de la première classe. Nous abordons à la fin les cas où le segment droit phrastique n'est pas un argument du prédicat décrit dans une phrase canonique.

\section{Structure prédicat-arguments du nom prédicatif}

La classe APE3 est définie par la structure canonique en (4a) ${ }^{3}$ :

(4) a. $\mathrm{N}_{0}$ (avoir + perdre) Dét $\mathrm{N}_{\text {préd }}(\mathrm{E}+$ Modif $)\left(E+\right.$ Prép $\mathrm{N}_{1}+$ Que $\mathrm{P}+$ de Vinf $)$

b. $=$ : Luc a un espoir raisonnable

c. $=$ : Luc a un désir fort de réussite sociale

$\mathrm{d}_{.}=$: Luc (a un + a perdu tout) (espoir + désir) de promotion

e. $=$ : Luc $(\mathrm{a}+\mathrm{a}$ perdu $)$ l'espoir (que son fils devienne avocat + de devenir avocat)

En (4a), l'élément « $E$ » dans le paradigme post-nominal indique que le second argument, qui peut être soit nominal ( $N_{\text {hum }}$ ou $N_{-h u m}$ ) soit phrastique (Que P, Vinf), n'est pas syntaxiquement obligatoire pour les $N_{\text {préd }}$ de cette classe, bien que la réduction s'accompagne souvent de l'ajout d'un modifieur (avoir un espoir raisonnable) ou d'un déterminant particulier (perdre tout espoir). En APE3, la préposition qui introduit le second argument est variable : à (capacité à), avec (difficulté avec), contre (argument contre), de (espoir de), devant (découragement devant), Loc (principalement dans et en, conviction dans) ${ }^{4}$, pour (intérêt pour), sur (doute sur), vers (élan vers), vis-à-vis de (réserve vis$\grave{a}$-vis de). Un même $N_{\text {préd }}$ peut se construire avec plusieurs prépositions (arrière-pensée de/ sur), et présenter d'autres différences dans les constructions, auquel cas l'entrée est dédoublée. Les déterminants sont variables entre zéro, un indéfini simple, un indéfini obligatoirement accompagné d'un modifieur, un partitif et le déterminant défini. 


\section{L'attribut phrastique du Nss et les arguments du nom prédicatif}

Les $N_{\text {préd }}$ sont toujours décrits dans une phrase assertive, qui admet des variantes autour de la même structure prédicat-arguments. Si un $N_{\text {préd }}$ accepte un complément phrastique comme argument (argument $N_{1}$, ici), une CCS peut exister parallèlement à la phrase canonique, et dans celle-là, le segment droit phrastique peut précisément être cet argument. Si tel est le cas, les deux types de phrases, canonique et spécificationnelle, constituent une classe d'équivalence de constructions, dans le sens où elles sont transformationnellement reliées, avec des différences interprétatives précisément déterminées et que nous explicitons ci-dessous. Schématisons :

(5) [Phrase canonique] $\mathrm{N}_{0}$ avoir Dét $\mathrm{N}_{\text {préd }}$ ((Prép ce) Que P + Prép Vinf)

$=:$ Luc a l'espoir d'acheter une nouvelle maison

[Construction spécificationnelle] Dét $\mathrm{N}_{\text {préd }}\left(\right.$ de $\left.\mathrm{N}_{0}\right)$ est $(\mathrm{Que} P+(\mathrm{de}) \text { Vinf })_{1}$

$=:$ L'espoir de Luc est d'acheter une nouvelle maison

11 Lorsqu'il s'établit entre les deux types de phrases une relation que l'on peut schématiser comme en (5), le segment droit de la construction spécificationnelle est un argument du $N_{\text {préd }}$, qui apparaît dans le segment gauche.

De ce point de vue, dans une CCS, le segment gauche est formé sur le $N_{\text {pré }}$ et son statut est celui du Nss. L'équivalence entre la phrase canonique et la phrase spécificationnelle en (5) permet d'interpréter la nature sous-spécifiée du nom comme le résultat de l'opération syntaxique qui consiste, d'un côté, à former un $S N$ prédicatif, et de l'autre, à en prédiquer une proposition, cette proposition étant, précisément, l'argument du $N_{\text {préd }}$. Cette particularité interprétative de la sous-spécification contraste nettement avec l'emploi du $S N$ de la même forme dans une phrase prédicative-attributive comme en (6) :

(6) L'espoir de Luc est risible

13 En (6), le $S N$ sujet est employé en tant que nom spécifié. Illustrons la différence entre Nss et nom spécifié : en (6), le locuteur est libre de préciser en apposition le contenu de ce à quoi réfère l'espoir de Luc, ce qui est exclu dans le cas de la CCS en (5):

(7) a. L'espoir de Luc, (qu'il achètera une nouvelle maison + d'acheter une nouvelle maison), est risible

b. *'espoir de Luc, (qu'il achètera une nouvelle maison + d'acheter une nouvelle maison), est (qu'il aura plus de confort + d'avoir plus de confort)

L'impossibilité observée en (7b) nous semble souvent utilisable comme critère formel appuyant l'intuition de Nss.

Il faut ajouter que l'autre argument des $N_{\text {préd }}$ ayant le fonctionnement de Nss est en général «humain». Il figure à l'intérieur du $S N$ formé sur le $N_{\text {préd }}$ à la suite de la réduction de la relative restrictive en avoir (cf. 4).

Le fait qu'un $N_{\text {préd }}$ possède la propriété d'avoir un argument phrastique n'est cependant pas en corrélation avec le fait qu'il fonctionne comme segment gauche d'une CCS. Il y a deux cas de figure : 1) la phrase canonique avec un complément phrastique est d'une acceptabilité assez basse (8a) et la CCS s'accepte très mal ; 2) la phrase canonique est acceptable mais la CCS ne l'est pas (8b) :

(8) a. ?? Luc a un grand acharnement à réaliser son projet ${ }^{5}$

?*L'acharnement qu'a Luc, c'est de réaliser son projet 
b. Luc a une grande capacité à enseigner

*Sa capacité est à enseigner

17 On peut citer dans cette classe des noms comme application (à), capacité (à), ferveur (à), hâte (à), incapacité (à), insistance (à), mauvaise grâce (à), construits avec la préposition à.

Par ailleurs, certains $\mathrm{N}_{\text {préd }}$ peuvent entrer dans une CCS avec un segment droit autre que le $\mathrm{N}_{1}$ décrit dans la table : bien que les $\mathrm{N}_{\text {préd }}$ embarras et émerveillement entrent naturellement dans des phrases spécificationnelles (9a-b), ils sont décrits avec un $\mathrm{N}_{1}$ non phrastique introduit par la préposition devant (9c) :

(9) a. Son embarras, c'est qu'il connaissait bien le protagoniste

b. Son émerveillement, c'est qu'il connaissait bien le protagoniste

c. Luc a eu un certain (embarras + émerveillement) devant ce spectacle

19 Les phrases $(9 a-b)$ ne sont donc pas à relier aux entrées d'APE3. Nous verrons ci-dessous qu'elles sont différentes de CCS ordinaires par le fait que le verbe copule est remplaçable par des verbes tels que venir (de), être dû (à).

\section{L'attribut phrastique de Nss correspond à un argument du $N_{\text {préd }}$}

20 Il existe grosso modo deux grandes classes parmi les exemples qui satisfont le schéma (5) : dans la première, le complément phrastique est le second argument du $N_{\text {préd }}$ et il n'a pas de relation de causalité avec le prédicat ; dans la seconde classe, il s'agit d'un argument prépositionnel du $N_{\text {préd }}$ et il assume le rôle sémantique de cause par rapport au prédicat.

(10) a. L'espoir de Luc, c'est qu'il sera élu président

(Quel est l'espoir de Luc?)

b. L'agacement (??de + pour) Luc, c'est qu'il y a trop de bruit dans son bureau

(??Quel est l'agacement (de + pour) Luc ?)

21 La CCS (10a) est de la première classe et (10b) de la seconde. Toutes les deux semblent répondre à la question en quel, donc assumant le critère de spécificationnalité, bien qu'en (10b), l'acceptabilité de la question en quel ne soit pas stable. En effet, contrairement à la première classe, (10b) est paraphrasable par des phrases où le rôle de la complétive vis-à-vis du $N_{\text {préd }}$ se précise grâce à des verbes autres que être, tels que (pro)venir (de) ou être dû (à), comme dans (11a): la proposition exprimée par le complément phrastique (segment droit) est considérée comme une "cause » de l'état des choses exprimé par le $S N$ prédicatif (segment gauche). La complétive n'est plus une simple Que P mais le fait Que P ou ce Que P :

(11) a. L'agacement de Luc ((vient du + est dû au) fait + (vient de + est dû à) ce) qu'il y

a trop de bruit dans son bureau

b. L'espoir de Luc ((vient du + est dû au) fait + (vient de + est dû à) ce) qu'il sera élu président

Si l'on paraphrase la CCS standard du type (10a) au moyen de verbes de causalité tels que venir (de) et être dû (à), le résultat (11b) n'est plus équivalent à (10a). 


\subsection{L'attribut phrastique n'est pas causal}

23 $N_{\text {préd }}$ déverbaux de cette classe (cf. 10a et 11b). Le complément phrastique du verbe regretter, ainsi que celui du $N_{\text {préd }}$ regret, peut d'ailleurs être considéré comme une réduction de la forme le fait (Que P + de Vinf) ou ce Que P :

(14) a. Paul regrette le fait (qu'il ait + d'avoir) dit une chose inappropriée

b. Paul a le regret de ce qu'il ait dit une chose inappropriée

Mais l'équivalence de (13b-c) peut n'être que pragmatique : si l'on pense à un scénario dans lequel Paul regrette d'avoir mangé de la viande sans le savoir, son regret peut être dû au fait qu'il est végétarien. Dans ce cas-là, le regret proprement dit et la cause du regret sont différents. Ce cas mérite d'être approfondi dans une étude ultérieure.

$$
\text { Prép }=: \grave{a}
$$

29 Très peu de $N_{\text {préd }}$ déverbaux en à acceptent d'entrer dans une CCS, et dans ce cas, l'infinitif est introduit par de et non par $\grave{a}$ :

(15) a. Luc (aspire + s'entête) à acheter une nouvelle maison

b. Son (aspiration + entêtement), c'est d'acheter une nouvelle maison

Certains acceptent mieux l'infinitif que la complétive dans la CCS, tandis que les constructions canoniques verbale et à $V_{\text {sup }}$ semblent accepter les deux types : 
(16) a. Luc (s'entête + s'empresse + a un certain (entêtement + empressement)) à (ce que tout soit prêt + tout préparer lui-même)

b. Son (entêtement + empressement) a été (de tout préparer lui-même + ?*que tout soit prêt)

31 Les phrases en (16) ont l'apparence d'une CCS, mais plusieurs restrictions sont observables : 1) la question en quel s'accepte mal (17a) ; 2) le temps ponctuel est préféré (comparer $17 \mathrm{~b}$ à $16 \mathrm{~b}$ et $17 \mathrm{a}$ à $17 \mathrm{c}$ )

(17) a. *Quel est son (entêtement + empressement) ?

b. ?*Son (entêtement + empressement) est (de tout préparer lui-même + que tout soit prêt)

c. ?*Quel a été son (entêtement + empressement) ?

d. *Son (entêtement + empressement) (vient du + est dû au) fait de devoir tout préparer lui-même causative de la proposition. Elle comporte des déverbaux reliés aux verbes acceptant un argument phrastique comme sujet dans une construction transitive : ce sont des verbes dits psychologiques (cf. 19b). Du point de vue de l'alignement entre les arguments et les compléments syntaxiques, la construction à $V_{\text {sup }}$ ressemble plutôt à la construction pronominale et/ou intransitive, lorsqu'elle est en usage, ou à la construction à participe passé adjectival (cf. 19c) :

(19) a. Luc a l'angoisse (qu'Ida le critique + de se faire critiquer par Ida)

b. (Qu'Ida le critique + Se faire critiquer par Ida) angoisse Luc

c. Luc ((s')angoisse + est angoissé) ((de ce) qu'Ida le critique + de se faire critiquer

par Ida)

d. Mon angoisse, c'est (qu'Ida me critique + de me faire critiquer par Ida)

5 À part le couple angoisser/angoisse, on peut compter dans cette classe des couples tels que agacer/agacement, confondre/confusion, contenter/contentement, etc.: ces noms entrent dans une CCS paraphrasable par les phrases à verbes de causalité, comme en (20b) :

(20) a. LE (angoisse + agacement + confusion + contentement +...) de Luc, c'est qu'il a été élu représentant

b. SON (angoisse + agacement + confusion + contentement +...) (vient du + est DU au)

fait qu'il a été élu représentant 
Dans ce travail, nous nous limitons à constater les faits suivants : certaines phrases copulatives ressemblent à des CCS sans en être vraiment, dans la mesure où la relation entre le segment gauche et le segment droit se précise autrement que par le verbe copule, notamment grâce à des verbes exprimant la causalité, comme venir de, être dî (à).

\section{L'attribut phrastique du Nss n'est pas un argument du nom prédicatif}

37 Nous présentons les cas où le segment droit Que $\mathrm{P} / \operatorname{Vinf}$ n'est pas identique à $N_{1}$. Les $N_{\text {préd }}$ de cette classe apparaissent dans une CCS mais ils sont différents des cas précédents 1) par le fait que le $N_{1}$ peut rester à l'intérieur du segment gauche et 2) que par conséquent, le segment droit phrastique est un élément supplémentaire par rapport à la structure prédicat-arguments définie en APE3. Voici un schéma de cette situation, où l'indice $s d$ est utilisé pour segment droit :

(21) $L E N_{\text {préd }}$ de $N_{0}$ Prép $N_{1}$ est (Que P + de Vinf) sd $_{\text {d }}$

Deux cas de figure s'observent: dans le premier, $N_{1}$ et le complément phrastique assument deux rôles sémantiques proches et entretiennent une relation sémantique que l'on appellera provisoirement relation de thème à $\operatorname{propos}^{7}$; dans l'autre, ils assument deux rôles sémantiques bien distincts.

\subsection{Thème et propos}

Le nom souvenir est à comparer au verbe se souvenir (de ce) Que P et le segment droit phrastique (22a) semble être identifiable au complément d'objet indirect du verbe (22b) ou du nom (22c-d) :

(22) a. Le souvenir de Luc est (que Paul a brillamment été reçu au concours + d'avoir acheté ce livre)

b. Luc se souvient ((de ce) que Paul a brillamment été reçu au concours + d'avoir acheté ce livre)

c. Luc a un bon souvenir (de ce + ?*E) que Paul a brillamment été reçu au concours d. Luc a le souvenir (que Paul a brillamment été reçu au concours + d'avoir acheté ce livre)

Or, ce $N_{\text {préd }}$ peut maintenir au sein du même $S N$ un complément de $N_{1}$ :

(23) Son souvenir de Mai 68 est (que Paris était en agitation permanente + d'avoir vécu les moments politiques les plus intenses de sa vie)

41 De $N$ en (23) peut correspondre, dans la phrase canonique, à un complément désignant le « thème » du « propos » exprimé dans la complétive. Il peut prendre d'autres formes, telles que à propos de $N$, sur $N$ :

(24) a. (À propos de + Sur) Mai 68, Luc a le souvenir que Paris était en agitation permanente

b. (À propos de + Sur) Mai 68, Luc se souvient que.../de...

Dans la structure canonique, de $N_{1}$ est difficilement compatible avec les compléments phrastiques dépendant directement du $N_{\text {préd }}$ et du verbe :

(25) * Luc (a le souvenir + se souvient) de Mai 68 de ce que Paris était en agitation permanente 

(26b), mais quand il fonctionne comme un argument verbal, on ne peut pas substituer à la préposition de d'autres prépositions (26a). La substitution semble possible, à première vue, dans une phrase nominale sémantiquement équivalente, mais ces deux types de compléments peuvent coexister dans la même phrase, plus facilement quand l'un des deux est détaché en tête (26c) :

(26) a. Luc se souvient (de + *sur + *à propos de) Mai 68

b. Luc a un beau souvenir (de + à propos de + sur) Mai 68

c. (À propos de + sur) Mai 68, Luc a un beau souvenir de Paris en grand désordre

(26c) militerait en faveur d'une interprétation en deux paradigmes distincts de ces deux types de compléments : d'un côté un argument ou un circonstant annonçant un thème général (à propos de + sur) $N_{2}$, et de l'autre un argument décrivant le propos (de $N_{1}$ ). Lorsque l'argument «propos » n'est pas précisé, le complément «thème » peut prendre la position de de $N_{1}$, neutralisant ainsi la distinction.

Parmi les $N_{\text {préd }}$ qui se construisent avec sur, la plupart se comportent comme souvenir: c'est le cas de idée (sur), illusion (sur), conviction (sur), certitude (sur), théorie (sur), etc.

\subsection{Autres cas de figure : preuve}

Le nom preuve, relié au verbe prouver, se définit en APE3 dans une structure telle que (27a), comparable à une phrase verbale comme (27b) où le prédicat sous-jacent est muni de deux arguments, l'un humain et l'autre phrastique :

(27) a. Luc a (une + la) preuve ((de ce) qu'il a été présent à la réunion + ?d'avoir participé à la réunion)

b. Luc prouve (qu'il a été présent à la réunion + avoir participé à la réunion)

Parallèlement à ces exemples, on observe une CCS du type suivant :

(28) La preuve qu'a Luc, c'est (qu'il a + d'avoir) laissé sa signature sur la feuille d'émargement

Or, du point de vue de l'interprétation, le segment droit dans (28) n'est pas celui qui se trouve en $N_{1}$ en $(27 \mathrm{a}-\mathrm{b})^{8}$. Le segment droit en (28) ne peut pas être considéré, malgré les apparences, comme la réalisation de l'argument $N_{1}$ du $N_{\text {préd }}$ preuve. Le fait que $N_{\text {préd }}$ et $N_{1}$ soient compatibles dans le segment gauche d'une CCS le démontre formellement :

(29) La preuve qu'a Luc de sa présence à la réunion, c'est (qu'il a + d'avoir) laissé sa signature sur la feuille d'émargement

En (29), qui diffère de (28) par l'ajout du complément de $N$ dans le segment gauche, ce $N$ assume le même rôle que $N_{1}$ en (27): $N_{1}=$ sa présence à la réunion = qu'il a été présent à la réunion. Cet ajout ne fait pas du segment gauche en (29) le nom spécifié, comme le montre le test d'apposition de la complétive :

(30) *La preuve qu'a Luc de sa présence à la réunion, qu'il a laissé sa signature, c'est que ses prises de parole sont sur le procès-verbal

Le segment droit de (29) n'est pas l'argument $N_{1}$ du $N_{\text {préd }}$ preuve, qui correspond à ce qui est à prouver, mais dénote la preuve même de $N_{1}$. Comme c'est le cas du $N_{\text {préd }}$ souvenir, pour réaliser ces deux compléments simultanément dans une phrase simple à $V_{\text {sup }}$, on aura recours à un moyen syntaxique comme l'apposition :

(31) Luc a la preuve de sa présence à la réunion $\left({ }^{*} \mathrm{E}+\right.$,) qu'il a laissé sa signature sur la feuille d'émargement 
51 À part le complément en de $N_{1}$, on peut citer des cas de ce type avec les compléments en pour, contre ou avec:

(32) a. Luc a un argument (pour + contre) la vente libre de cannabis

b. L'argument qu'a Luc (pour + contre) la vente libre de cannabis, c'est que ça augmente la richesse de certains

c. Luc a un (intérêt + ennui) avec cette affaire

d. L'(intérêt + ennui) qu'a Luc avec cette affaire c'est qu'elle est très lucrative

\section{Conclusion}

Dans la littérature, peu d'attention a été accordée à la nature prédicative du nom sousspécifié, et encore moins au statut argumental ou non du complément phrastique dans une CCS. Considérer la question du point de vue de l'ensemble prédicat-arguments permet de donner un critère formel de reconnaissance du nom sous-spécifié dans le cadre de CCS. On a observé, à ce propos, qu'un $N_{\text {préd }}$ peut entretenir des relations avec plus d'un complément phrastique, dont un seulement est généralement considéré comme son argument. L'autre complément est accessoirement intégré dans une phrase canonique par le biais d'une apposition, tandis qu'en CCS, il joue le rôle du segment droit. Par ailleurs, ce travail permet également de situer au niveau du segment gauche un autre argument peu évoqué dans les études précédentes: un argument non phrastique du Nss. L'étude a enfin révélé l'existence de plusieurs types de constructions spécificationnelles : chacun possède une paraphrase différente des autres, laquelle met en évidence une interprétation particulière.

Ce travail préliminaire est à étendre à la totalité des tables du lexique-grammaire de $N_{\text {préd }}$, qui s'enrichiront de nouvelles structures phrastiques.

\section{BIBLIOGRAPHIE}

Boons, J.-P., Guillet, A. \& Leclère, C. (1976a). La structure des phrases simples en français. Constructions intransitives. Genève-Paris : Droz.

Boons, J.-P., Guillet, A. \& Leclère, C. (1976b). La structure des phrases simples en français. Classe de constructions transitives. Rapport de recherches 6 . Laboratoire d'automatique documentaire et linguistique. Université Paris 7.

Giry-Schneider, J. (1978). Les nominalisations en français. L'opérateur faire dans le lexique. Genève-Paris : Droz.

Giry-Schneider, J. (1987). Les prédicats nominaux en français. Genève-Paris : Droz.

Guillet, A. \& Leclère, C. (1992). La structure des phrases simples en français. Constructions transitives locatives. Genève-Paris : Droz.

Gross G. (1989). Les constructions converses du française. Genève-Paris : Droz.

Gross, M. (1975). Méthodes en syntaxe. Paris : Herman. 
Harris, Z. S. (1965). “Transformational theory”. Language 41, 363-401.

Labelle, J. (1974). Etude de constructions avec opérateur avoir (nominalisation et extensions). Thèse de 3ème cycle. Université Paris 7.

Legallois, D. (2006). « Quand le texte signale sa structure : la fonction textuelle des noms sousspécifiés ». Corela HS-5. (http://corela.revues.org/1465)

Legallois, D. (2008). « Sur quelques caractéristiques des noms sous-spécifiés ». Scolia 23, 109-127.

Legallois, D. \& Grea, P. (2006). « L'objectif de cet article est de... Construction spécificationnelle et grammaire phraséologique ». Cahiers de praxématique 46, 161-186.

Nakamura, T. (2009). «Sur les arguments sémantiques du verbe expliquer et leur réalisation syntaxique ». Lingvisticæ Investigationes 32, 187-199.

Nakamura, T. (2017). « Extensions transitives de constructions spécificationnelles ». Langue française 194, 69-84.

Vivès, R. (1983). Avoir, prendre, perdre : constructions à verbe support et extensions aspectuelles. Thèse de 3ème cycle. Université Paris 8.

\section{NOTES}

1. Le lexique-grammaire du français est un programme de recherche qui a pour objectif de construire des bases de données lexicales et syntaxiques, et dont la charpente théorique est bâtie sur la théorie distributionnelle et transformationnelle de Z. S. Harris (Harris 1965, par exemple). L'unité de base est un cadre de phrase dite simple, avec une forme prédicative (verbale, nominale ou adjectivale) particulière, munie de tous ses arguments. Pour chaque phrase simple, l'applicabilité d'un ensemble d'opérations syntaxiques (appelées transformations) est vérifiée et le résultat est présenté dans une matrice appelée "table de lexique-grammaire ». Le projet a débuté par la construction de classes de phrases simples comportant au moins une complétive et/ou une infinitive (Gross 1975) et il a été étendu à la description de tous les cadres de phrases verbales (Boons, Guillet et Leclère 1976a, b ; Guillet et Leclère 1992). D'autres formes prédicatives ont été décrites avec les mêmes principes et le lexique-grammaire s'est étendu à d'autres langues.

2. Un nom peut être prédicatif dans le sens où il est muni de ses propres arguments et peut constituer le noyau d'une phrase simple. Le nom prédicatif a besoin d'une béquille grammaticale pour fonctionner comme prédicat d'une phrase: il se combine avec un ou plusieurs verbes appelés « verbes supports " qui apportent dans la phrase les informations de temps, d'aspect et de mode. Les compléments de tels verbes ne sont pas leurs arguments, mais ceux du $N_{\text {préd. }}$. Les noms prédicatifs ont été étudiés selon les principes du lexique-grammaire : différents cadres de phrases simples ont été définis par leurs structures d'arguments et par différents verbes supports (faire, Giry-Schneider 1978, 1987 ; avoir, Labelle 1974 ; avoir, prendre et perdre, Vivès 1983 ; donner et recevoir, Gross 1989...). Les propriétés distributionnelles et syntaxiques ont été vérifiées pour chaque type de cadre et le résultat est également représenté dans des tables du lexiquegrammaire. Dans le cadre du lexique-grammaire, Nakamura (2017) a étudié la construction $N_{0}$ avoir pour Nss (Que P + de Vinf).

3. Les parenthèses désignent un paradigme et ses éléments au choix sont séparés par le signe «+». Dans la structure canonique, le premier argument du $N_{\text {préd }}$ est $N_{0}$, le deuxième $N_{1}$. Le symbole « $E$ » indique que le paradigme peut rester vide. Le symbole «=:» signifie la réalisation d'un schéma syntaxique. 
4. L'abréviation Loc désigne les prépositions qui introduisent ordinairement les compléments locatifs.

5. Par rapport au verbe support avoir, acharnement (et également, application, empressement, entêtement, etc.) se construit plus naturellement avec le verbe support mettre, formant un équivalent du verbe s'acharner :

Luc met de l' (application + acharnement + entêtement...+$)$ à réaliser son projet

Luc (s'applique + s'acharne + s'entête +...) à réaliser son proje

Ce nom s'observe également dans une construction en il y $a$, précédé du déterminant indéfini :

Il y a un acharnement de la part des migrants à tenter leur chance

6. Pour rendre la description compacte, les mots ou expressions morphologiquement variables sont représentés par la forme masculine singulier notée en majuscules: $L E$ est à interpréter comme le, la ou les, selon les noms dont il dépend.

7. Nous utilisons les termes «thème » et "propos " pour caractériser la relation entre les deux compléments (nominal et phrastique) qui peuvent s'articuler autour d'un même $N_{\text {préd }}$. Il ne s'agit pas $\mathrm{du}$ « thème » ni du « propos » de la phrase en entier au sens informationnel.

8. Le nom explication, relié au verbe expliquer, est comparable au nom preuve, cf. Nakamura (2009).

\section{RÉSUMÉS}

Les noms sous-spécifiés (Nss), qui peuvent apparaitre dans une construction spécificationnelle (1), de la forme Leur ambition a été d'innover, sont par ailleurs souvent des prédicats régissant des arguments, dans une phrase élémentaire (2) de la forme Ils ont eu l'ambition d'innover, où Nss est le prédicat accompagné d'un verbe support et de ses arguments $N_{0}$ et $N_{1}$. Nous avons étudié des noms entrant à la fois dans les deux constructions (1) et (2) avec le même sens, et trouvé trois cas de figure. Avec des noms tels que ambition, (1) et (2) ont presque le même sens. Dans d'autres entrées, telles que souvenir et preuve, les deux constructions ne sont pas équivalentes : l'attribut phrastique de (1) est absent de (2), tandis que l'argument $N_{1}$ de (2) peut apparaitre comme complément de nom du Nss sujet dans (1), et spécifie souvent (c'est le cas pour souvenir) le thème du propos contenu dans l'attribut phrastique de (1). Enfin, d'autres noms tels que embarras ne sont pas des Nss typiques car l'attribut phrastique de (1) y joue un rôle causal ; il n'a pas le même contenu que $N_{1}$. Ainsi, dans les types souvenir/preuve et embarras, (1) est à analyser comme une combinaison de plusieurs phrases élémentaires.

Underspecified nouns (Nss), those that can appear in a specificational construction (1) of the form Leur ambition a été d'innover "Their ambition was to innovate", are often also predicates governing arguments in an elementary sentence (2) of the form Ils ont eu l'ambition d'innover "They had the ambition to innovate", where Nss is the predicate accompanied by a support verb and its arguments $N_{0}$ and $N_{1}$. We studied nouns entering in both constructions (1) and (2) with the same meaning, and found three situations. With nouns such as ambition, (1) and (2) have almost the same meaning. With other entries, such as souvenir "memory" and preuve "proof", the two constructions are not equivalent: the sentential predicative of (1) is absent from (2), whereas the $N_{1}$ argument of (2) can appear as a prepositional complement of Nss in (1), and often (as with souvenir) specifies the theme of the content of the sentential predicative of (1). Finally, nouns such as embarras "embarrassment" are not typical Nss because the sentential predicative of (1) 
has a causal role; it has not the same content as $N_{1}$. Thus, in the mémoire/preuve and embarras types, (1) seems to involve several elementary sentences.

INDEX

Mots-clés : nom sous-spécifié, lexique-grammaire, nom prédicatif

Keywords : underspecified noun, lexicon-grammar, predicative noun

\section{AUTEURS}

\section{ÉRIC LAPORTE}

LIGM, Université Gustave Eiffel, CNRS, ESIEE Paris

\section{CHRISTIANE MARQUE-PUCHEU}

Lettres Sorbonne Université, STIH (EA 4509)

\section{TAKUYA NAKAMURA}

LIGM, Université Gustave Eiffel, CNRS, ESIEE Paris 